\title{
Control System Design of Magneto-rheoloical Damper under High-Impact Load
}

\author{
Bucai Liu \\ College of Mechanical Engineering, University of Shanghai for Science and Technology \\ 516 Jun Gong Road, Shanghai 200093, China \\ Tel: 86-159-2118-3769 E-mail: liubucai043@163.com \\ Jinjie Chen \\ College of Mechanical Engineering, University of Shanghai for Science and Technology \\ 516 Jun Gong Road, Shanghai 200093, China \\ Tel: 86-21-5527-1607 E-mail: jinjiechen@yahoo.com.cn
}

Received: August 10, 2011

Accepted: August 30, 2011

doi:10.5539/mas.v5n5p253

\begin{abstract}
In recent years, the performance requirements of the mechanical and electrical products are increasingly, how to improve those products' impact resistant ability while in the environment of high impact is become very important. Because of the traditional damper device could not do the real-time adjust about the damping force during the high impact process, in this paper, a magneto-rheological damper is applied to cushion the high-impact load. And the control current, passing into the electromagnetic coil of damper, is changed by designing the delay and fuzzy PID control algorithm in real time, which achieve an effective buffering control of high-impact load. The whole control method is based on TMS320F2812 to achieve. Experimental results show that it can significantly improve the dynamic performance of the damper control system by real-time precise control of the delay and fuzzy PID control algorithm, increase the stability of the damping process.
\end{abstract}

Keywords: High-impact, Magneto-rheological damper, DSP, Delay and fuzzy PID control

\section{Introduction}

In recent years, the performance requirements of the mechanical and electrical products are increasing, high impact resistant ability is becoming one of the important marks to measure the performance of products, how to improve the impact resistant ability while in the environment of high impact is becoming very important. (Zhang, Wang \& Qian, 2008). Therefore, the control system design of magneto-rheological damper under high-impact load is very important.

Traditional damper device could not do the real-time adjust about the damping force during the high impact process, compared with traditional damper, the magneto rheological damper which uses the magnetic rheological liquid as the medium, and its output damping force is controlled by working current, besides, it has the advantages such as the structure is simple, the power consumption is low, can provide high damping force, the range of damping force is adjustable, fast response speed and other characteristics. (Hou, 2006). In addition, use a robust time delay fuzzy PID control method to improve the dynamic performance of the damper control system.

According to the experimental results, we can find that applying time delay fuzzy PID control can improve the peak of damping force, enhance the stability of the impact, significantly better than the fixed current control method. The buffer process can well realize the performance optimization of the displacement and force curve, optimize dynamic performance of the system.

\section{Magneto rheological damper and its characteristics}

Because the high impact process is a process of unidirectional long stroke, in this paper, the single rod long stroke magnetic rheological damper was adopted, and the pressure compensation mechanism was abolished. The damper structure is shown in Figure 1, including cylinder, piston rod, piston head, a guide rod, seals, magnetic circuit, front back and other parts. 
The piston rod is connected with the fixed base; damper cylinder axis is connected with the impact mass block. During the buffer process, the mass block and the cylinder along with the guide rail move to the right, then the relative motion which between piston rod and cylinder produces the damping force. Magneto rheological damper buffers the high impact load, converts to small damping force on the fixed base. From the functional point of view, the role of magneto rheological damper is buffering the process to consumption and storage part of energy which causes by the high-speed impact, control the stress and movement rules, and control the buffer movement in appropriate length range.

\section{The hardware design of the control system}

The control system includes actuator, magneto rheological damper, signal sensing system, power driver circuit and DSP (TMS320F2812). System structure diagram is shown in Figure 2.

When the magneto rheological damper executing the buffering process, sensor collect the displacement signal, speed signal, force signal and the feedback current signal of the actuating mechanism in real-time, the DSP controller using AD sampling correction function to do real time sampling of these signals, and using these signals as the input signal of control algorithm. DSP use the event manager timer 1 trigger AD sampling correction, after each sampling cycle, the DSP will update the compare register CMPR1 according to the control algorithm results of input signal. The comparison unit of the event manager EVA will output duty ratio to change PWM (pulse width modulation) in time according to the result of CMPR1. Then, use the optical chip TLP250 amplification the PWM signals, and output them to the PMOS tube, to make the power driver circuit work.

\section{The software design of the control system}

The software design mainly relates to signal sampling, control algorithm, output of PWM signal and SCI transmission. Software programming mainly consists of main program, system initialization, interrupt vector initialization procedure, the $\mathrm{AD}$ sampling correction interrupt response procedure, control algorithm processing procedures, EV interrupt response procedure and SCI data transmission. The software flow diagram is shown in Figure 3. DSP control program use the interrupt vector method to achieve: start timer count T1, wait for interrupt, set the value of cycle timer register T1PR to determine the AD sampling cycle and the output frequency of the PWM, when completed a interrupt, system return to the main program to wait for the next interrupt.

\section{Control algorithm of magneto rheological damper under high impact condition}

Under high impact condition, when magneto rheological damper is reducing the force which impact load transmit to the installation site, will increase the displacement in the buffer process. But, to reduce the length of the displacement, the force of the damper will increase. Therefore, in the buffer process, damper force and displacement is a pair of contradiction variable. In this paper, the design of the control algorithm is to balance the damper force and displacement, to realize the damper force and displacement curve as painless as possible. Besides, because of the whole control system running in high speed impact condition, the whole process time is about $130-140 \mathrm{~ms}$, so the control algorithm should ensure the real time. Through the experimental verification, applying time delay fuzzy PID control algorithm can improve the dynamic performance of the whole system in the impact process, and can satisfy the real time requirement.

\subsection{Time delay control}

Displacement signal, the force signal and the damper chamber pressure signal are used as the criterion of the dynamic performance of damper. The experimental results show that if applying current to the damper at the beginning of the impact loading, it will cause the damper force signal peak and intraluminal pressure signal peak increase sharply, the dynamic performance of the whole system will be influenced. While applying current to the damper after time delay of the impact loading, the damper force signal peak and intraluminal pressure signal peak may be markedly improved.

Therefore, we can take the displacement signal as the reference signal for the system, use delay control, applying control current to the damper after the impact of the force peak; it can improve the dynamic performance of the damper.

\subsection{Fuzzy PID control}

In order to realize the fuzzy PID control, the system uses two levels of closed loop. The fuzzy PID control system block diagram is shown in Figure 4. The outer ring is design to correction the adjustable Coulomb resistance $F_{t}$ through fuzzy rule, the inner ring is design to correction the current of damper coil electromagnetic loop through fuzzy PID.

(1) The outer ring is to realize the feedback control of adjustable output Coulomb resistance $F_{t}$, through the 
feedback of the damping force signal and the speed signal real-time to calculate $\mathrm{F}_{\mathrm{t}}$, and compared with ideal $\mathrm{F}_{\mathrm{tr}}$, using fuzzy rules for feedback control, to determine the ideal value of the output control current.

This paper set the ideal recoil resistance $\mathrm{F}_{\mathrm{Rr}}$ as a constant, according to the ideal damping dynamic process to design the ideal coulomb resistance curve $\mathrm{F}_{\mathrm{tr}}$.

Step one

Through the impact recoil differential equations to calculate the ideal recoil velocity $\mathrm{V}_{\mathrm{rr}}$ and the ideal recoil displacement $\mathrm{S}_{\mathrm{rr}}$ :

$m_{h} \frac{d v_{r r}}{d t}=F_{p t}-F_{R r}$

$\mathrm{m}_{\mathrm{h}---- \text { Recoil mass, } \mathrm{kg}}$

$\mathrm{V}_{\text {rr }}$---- Ideal recoil velocity, $\mathrm{m} / \mathrm{s}$

$\mathrm{F}_{\mathrm{pt}}$----The actual role shock load, $\mathrm{N}$

$\mathrm{F}_{\mathrm{Rr}}----$ The ideal recoil resistance, $\mathrm{N}$

Step two

Determining the ideal Coulomb resistance $\mathrm{F}_{\text {tr }}$.

$F_{\tau r}=F_{R r}-a v_{r r}{ }^{n}-F_{f}$

$\mathrm{F}_{\text {tr }}$----Ideal Coulomb resistance, $\mathrm{N}$

a---- coefficient, can obtain from the system parameter, in this system, $a=300$

$\mathrm{n}$---- Fluid behavior index, $\mathrm{n}=1.6$

$\mathrm{F}_{\mathrm{f}}$---- The friction between the guide rail and the recoil device, $\mathrm{N}$

Step three

Obtain the feedback parameters adjustable Coulomb resistance $\mathrm{F}_{\mathrm{t}}$ :

$F_{\tau}=F_{M R D}-a v_{r}{ }^{n}$

$\mathrm{F}_{\mathrm{MRD}^{----}}$Test recoil resistance value, $\mathrm{N}$

$\mathrm{V}_{\mathrm{r}---}$ Test recoil velocity value, $\mathrm{m} / \mathrm{s}$

Besides, the control algorithm $\mathrm{aV}_{\mathrm{r}}^{\mathrm{n}}$ is adopted in form in the hardware realization, to ensure the real time of the control.

Last, use of the difference value e that about the ideal Coulomb resistance $\mathrm{F}_{\text {tr }}$ and adjustable Coulomb resistance $\mathrm{F}_{\mathrm{t}}$, using fuzzy control rules to judge the size of theory control output current.

(2)The inner ring is to realize the fuzzy PID correction about the current of the damper coil electromagnetic loop, to output the accurate control current and reduce the response time of the coil. Its principle diagram is shown in Figure 4.

In order to improve the output precision of control current, comparison the calculated current value and the actual feedback current value, calculate the value of error, using the fuzzy PID control then output the new control current. Use the two inputs three outputs fuzzy controller, the error signal e and the change rate of current error ec signal as the input signals of the fuzzy controller, to setting the PID parameter $\mathrm{Kp}, \mathrm{Ki}, \mathrm{Kd}$.

Fuzzy PID control fuzzy rules can obtain through off-line calculation, produce in tabular form, this can reduce the amount of computation, ensure the real-time control.

\section{The result of impact test}

Figure 5 is the effect of time delay control, the impact process is approximately $130-140 \mathrm{~ms}$. The solid line represents use the fixed current control strategy to create the displacement and force curve, the dotted line represents use the time delay fuzzy PID control. As can be seen from the graph, using time delay fuzzy PID control, the damping displacement increased from $85 \mathrm{~mm}$ to $135 \mathrm{~mm}$, growth of $59 \%$; damping force peak is significantly improved, from $9600 \mathrm{~N}$ down to $2400 \mathrm{~N}$, fall $75 \%$; compared to the fixed current curve, the time delay fuzzy PID control method is smoother during the whole process.

According to the experimental results, it is not difficult to find that applying time delay fuzzy PID control can 
improve the damping force peak, enhance the stability of the impact, significantly better than the fixed current control method. The buffer process can well realize the performance optimization of the displacement and force curve, optimize dynamic performance of the system.

\section{Conclusions}

Because of the traditional damper device couldn't realize the real-time adjust about the damping force during the high impact process, in this paper, the magneto rheological damper which applicable to high impact was adopted, and designed the delay fuzzy PID control algorithm. Through the impact test, results show that single bar long-stroke magnetic rheological damper can buffer the high impact load well, and time delay fuzzy PID control method can significantly improve the system dynamic performance while under high impact damping condition, increase the stability of the damping process. The DSP chip in the system has fast operation speed, can meet the requirement of real-time control.

\section{References}

Hou,B.L.(2006). Modeling and Analysis of Magnetorheological Dampers under Impact Load. Chinese Journal of Mechanical Engineering, 42(4):174-178.

Li,Y.C.,Wang,J.,\&Qian,L.F.(2006). Magnetorheological Technology and Its Latent Applications in MilitaryField. Journal of ballistics, 18(3):68-8.

Liu,Y.M., Faramarz, G., Cahit, E., \&Sinan, K.(2004). Experimental study on fuzzy skyhook control of a vehicle suspension system using a magneto-rheological fluid damper. Smart Structures and materials,338-347.

M,A.(2008). Experimental analysis of magnetorheological dampers when subjected to impact and shock loading. Communications in Nonlinear Science and Numerical Simulation,1978-1985.

Michael,J.C., Gregory, D.B., \& Richard, D.(2003). Fuzzy Logic Control Algorithms for MagneShock Semi-Active Vehicle Shock Absorbers: Design and Experimental Evaluations. Smart Structures and materials,577-588.

Yao, J., Li, Y.Q., Hou, Y.F., \& Pan, M.H.(2009). Asynchronous Motor based on DSP Adaptive Fuzzy PID Control Design. Instrumentation customer,05(29):50-52.

Zhang, L.J., Wang, J.,\& Qian, L.F. (2008).MR Dampers under Impact Load Dynamic Characteristics Test and Analysis. Acta ARMAMENTARII, 29(5):532-536.

Zhang,W.N.(2004). Texas Instruments Incorporated.TMS320C28x DSP CPU and Peripherals. Beijing: Tsinghua University press, (Chapter 3).

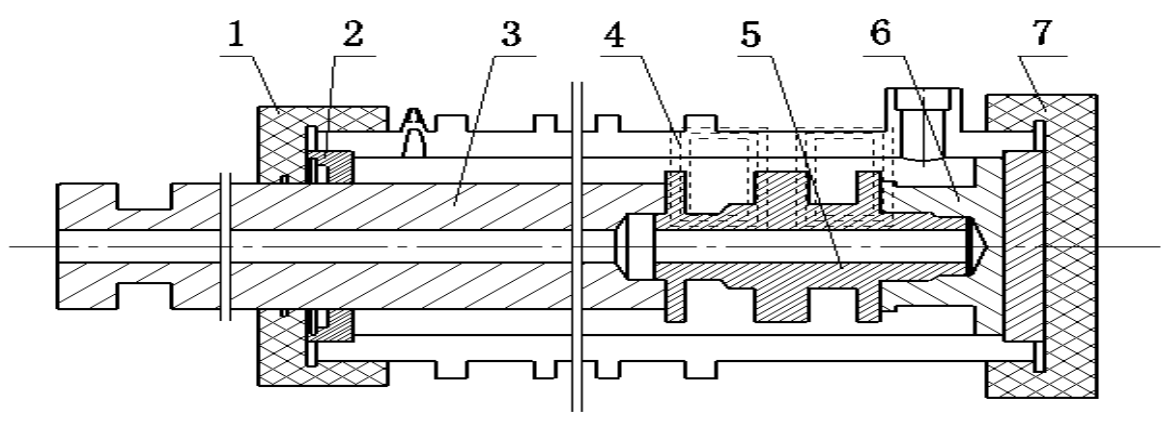

1-Front cover 2-Sealing plug 3-Piston rod 4-Magnetic circuit 5-Piston head 6-Guide rod 7-Rear cover

Figure 1. Structure Diagram of Magneto Rheological Damperc 


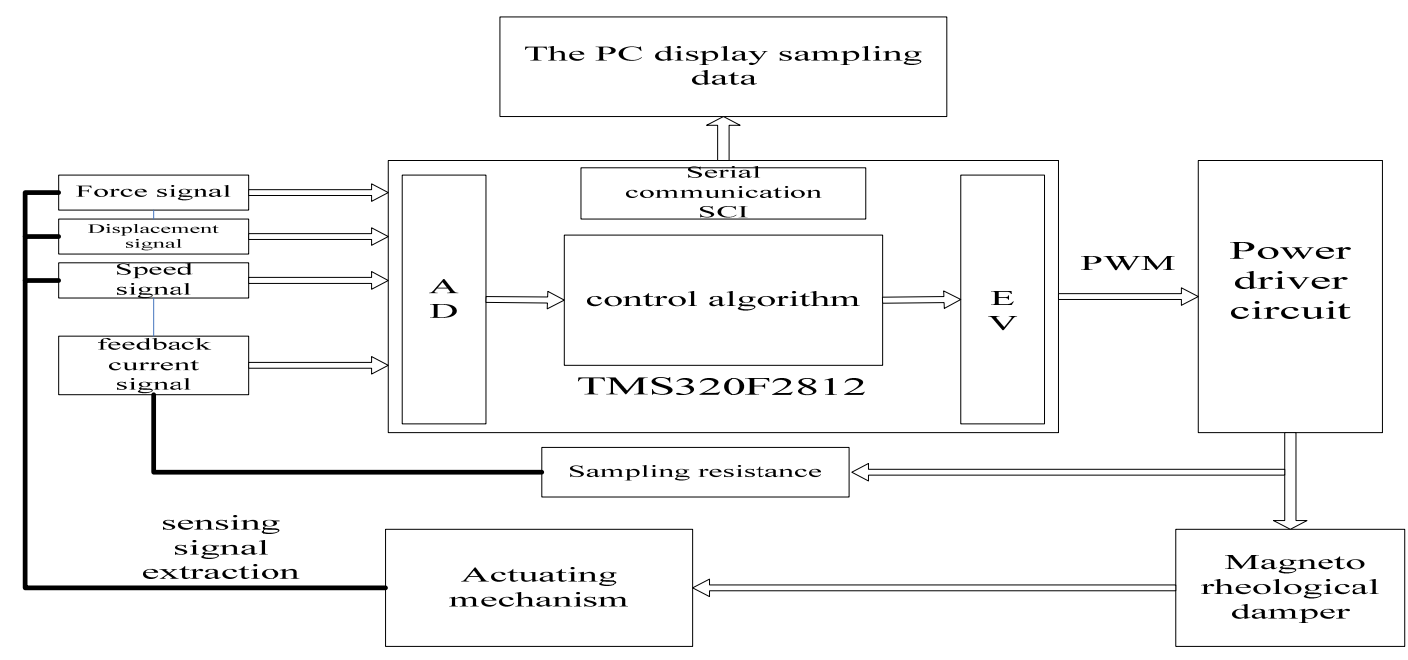

Figure 2. Control System Structure Diagram of Magneto Rheological Damperc

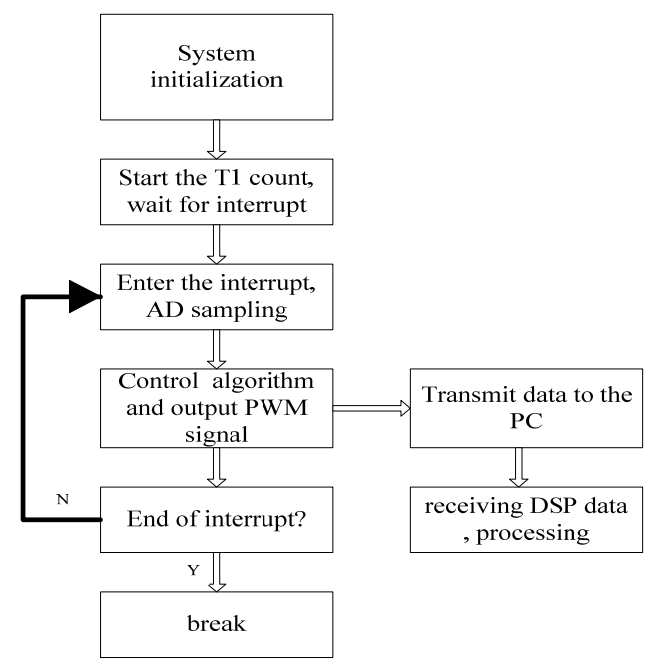

Figure 3. Flowchart of Control System

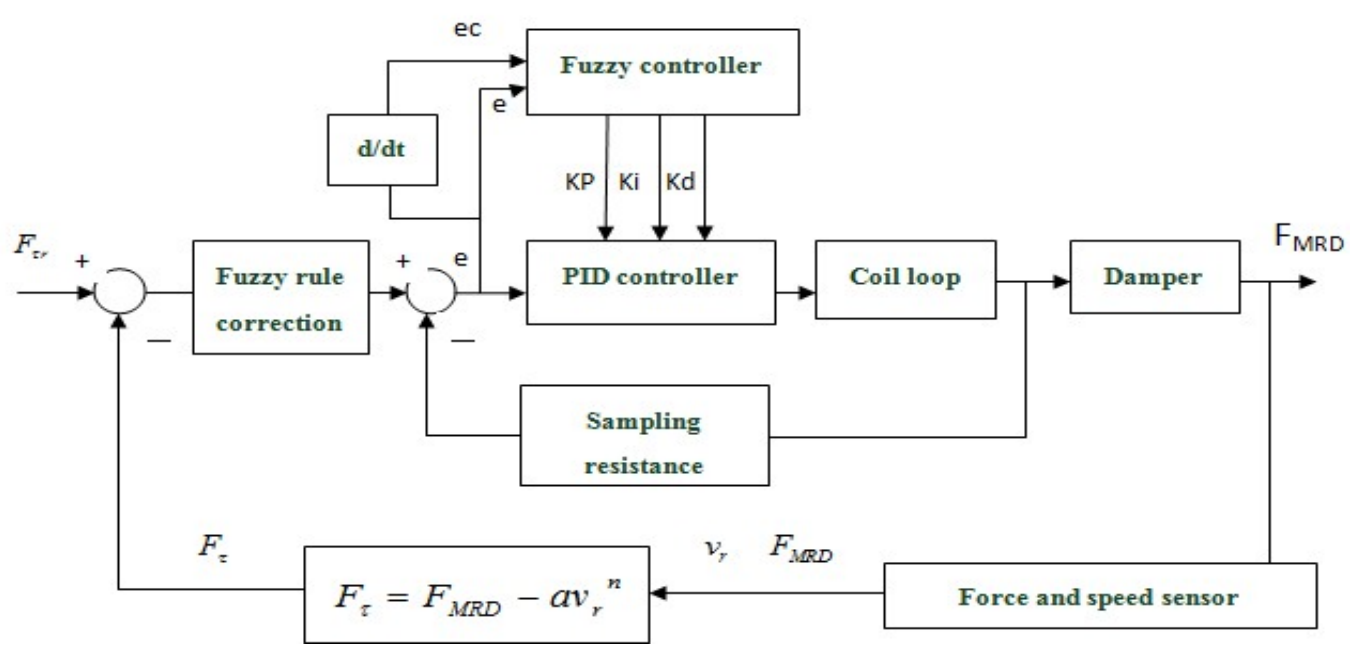

Figure 4. Block Diagram of Fuzzy PID Control 


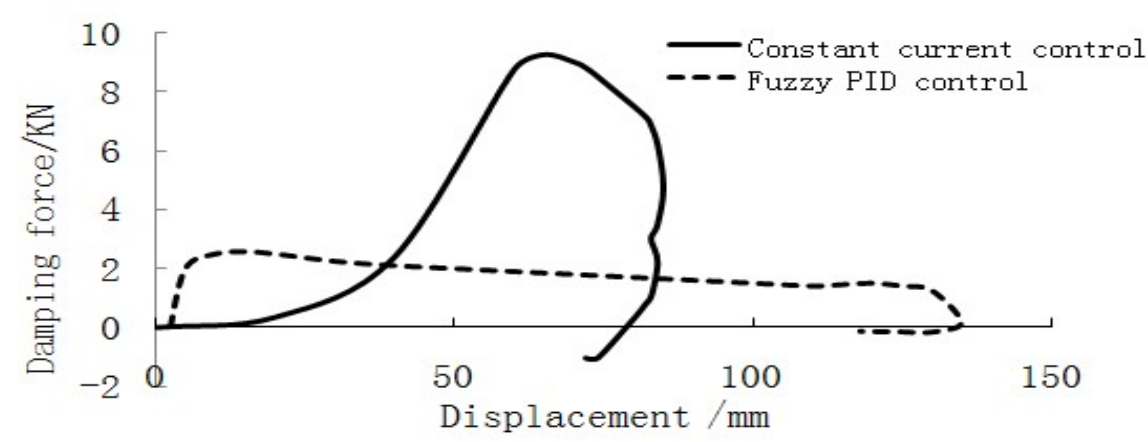

Figure 5. Experimental Results of Impact Load 